\section{Role of Ultrasound in the Imaging of Parotid Swellings}

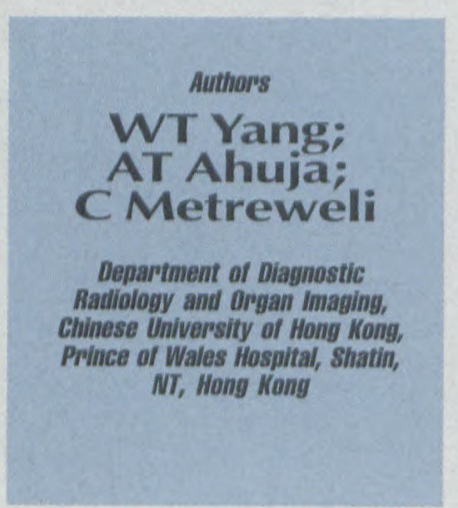

\begin{abstract}
This pictorial essay details

anatomy of the normal

parotid as well as a

spectrum of intra- and

extraparotid pathology.

Extraparotid lesions which

mimic a parotid swelling

are well characterised on

ultrasound. In addition,
\end{abstract}

sonographic features of

intraparotid neoplasms,

inflammation and infection

are also illustrated. We

recommend ultrasound as

a useful first line approach

in the evaluation of parotid

disease, and also as an

aid to guided biopsy of

both intra- and

extraparotid masses.

\section{Introduction}

When imaging a parotid swelling, it is necessary to determine if the lesion is intra- or extraparotid. Intraparotid lesions may be inflammatory or neoplastic, benign or malignant, solitary or multiple and lie superficial or deep to the facial nerve. Ultrasound (US) is particularly sensitive in the detection of focal lesions in the superficial portion of the gland. US guided fine needle aspiration and cytology (FNAC) may furtherenhanceits ability to differentiate between intra/extraparotid lesions or predict the malignant/benign nature of a tumour. The main disadvantage of parotid US is in the evaluation of deep lobe lesions, parapharyngeal extension and skull base involvement.

\section{Method}

Real timescans of the parotid region were obtained using either a $5 \mathrm{MHz}$ or $7.5 \mathrm{MHz}$ linear array or $10 \mathrm{MHz}$ mechanical sector transducer with inset water bath (Aloka 650). Imaging of superficial parotid lesions was improved by using the linear $5 \mathrm{MHz}$ transducer with 4 inch by 4 inch $3 \mathrm{Mstand-offpad,}$ as this placed the lesion in the optimal focal zone of the transducer. Transverse and longitudinal (coronal) scans were performed with the patients supine and head turned away from the side being imaged. Transverse images were obtained with the transducer perpendicular and inferior to the ear lobe. When performing longitudinal scans, care was taken to angulate the transducer anteriorly in order to search for lesions in the tail of the parotid that would be obscured by the mandibular ramus.

\section{Normal US \\ Anatomy of the Parotid}

The parotid gland is the largest of the salivary glands and is triangular in configuration. It demonstrates smooth, homogenous and fine bright echoes on US. Its major anatomical land marks on axial imaging are the masseter muscle

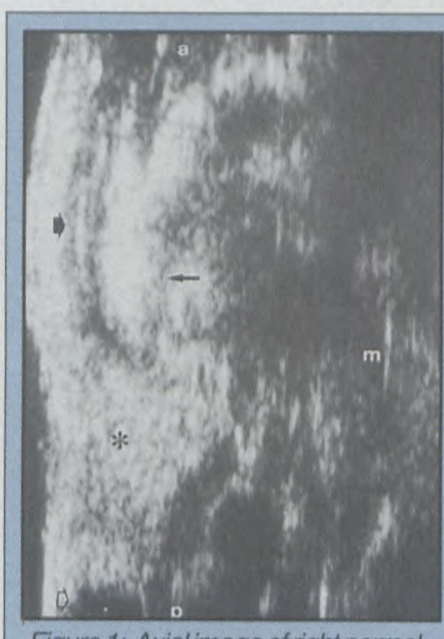

Figure 1: Axial image of night nomal parotid demonstrating anatomical relations. Parotid gland(asterisk), massetermuscle (shortamow), mandibular ramus (longamow), stemocleidomastoidmuscle(open shortarrow).

and mandibular ramus anteriorly, the sternocleidomastoid muscle and mastoid process posteriorly, and the carotid sheath medially (Figure 1). Superiorly it extends to just below the level of the zygomatic arch. The facial nerve forms the boundary for the surgical division of the parotid gland into superficial and deep lobes. The nerve is however not visible on US, and its position is inferred as it parallels the course of the Stenson's duct through the gland (Figure 2). The retromandibular vein (branch of the external carotid vein) runs through the deep portion of the gland (Figure 3).

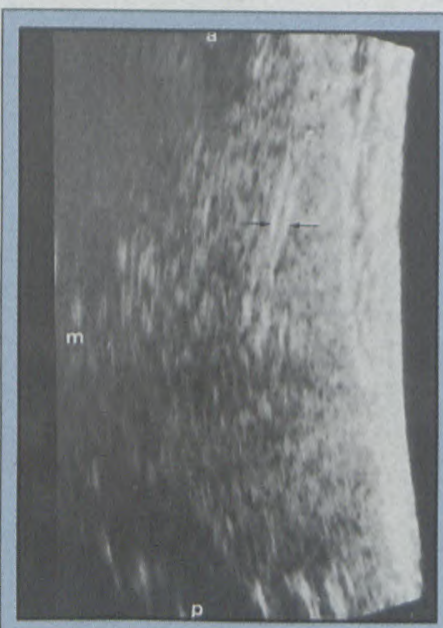

Figure 2: Transverse (axial) image of left parotid shows Stenson's ductas two parallelechogenic lines (amows) coursing through the parotid. 


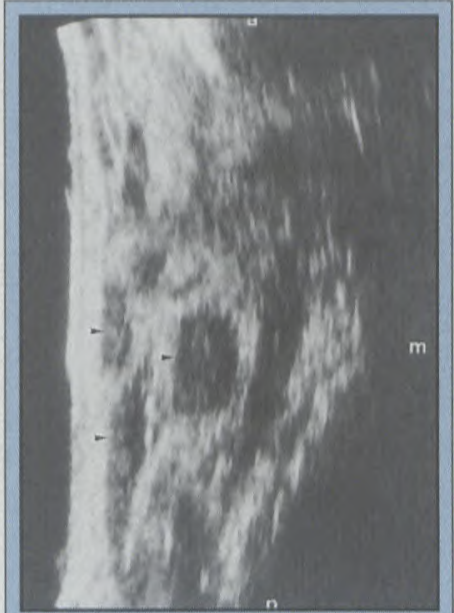

Figure 3: Longitudinal (coronal) image on the right demonstrates the

retromandibular vein (arrows) in the

deep portion of the parotid.

Intraparotidlymph nodes are normally not visible or very sparse and small except in children. There may be from 10 to 20.They drain into the superficial and deep cervical chains'. The accessory

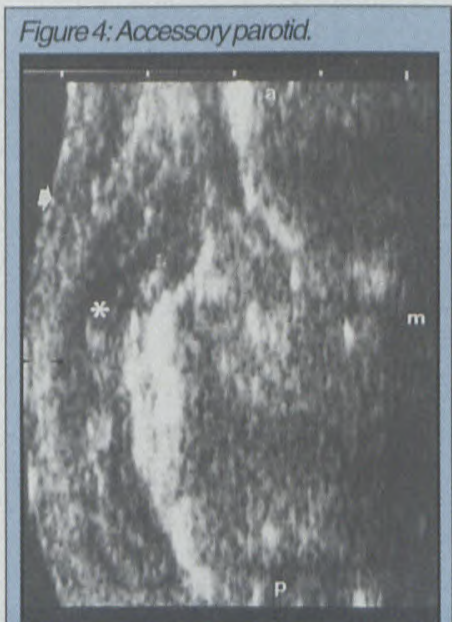

A. A sliver of parotid tissue (short arrow) is notedadjacent to Stenson's duct (amowheads) andmasseter muscle (asterisk) onaxialUS.

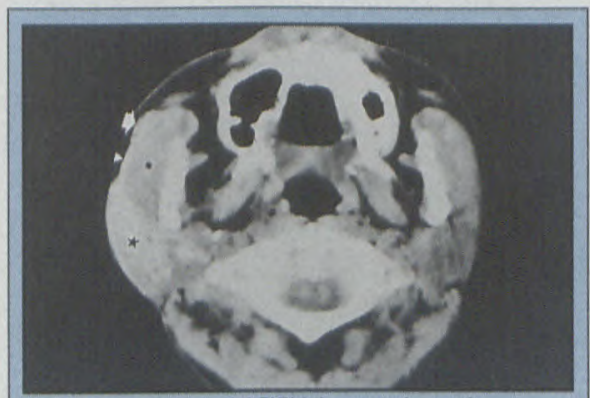

B. ComespondingaxialCT/sialogram shows the accessory parotid(shortamow) superficial to the Stenson's duct (anrowhead) andmassetermusde (asterisk), separate from the main parotid gland(star). parotid gland is found adjacent to the parotid duct separate from the main body of the gland (Figures 4A,4B) and should not be mistaken for pathology.

\section{Extraparotid lesions}

Whether a parotid swelling is of intra- or extraparotid origin is important to the surgeon as the surgical approach differs for both lesions. Intraparotid lesions require a transparotid approach with localisation and control of the facial nerve, whilst extraparotid lesions may be approached perorally or via the submandibular region with no attempts at facial nerve control.

Masses arising from outside the salivary gland which mimic a parotid swelling show no rim of parenchyma surrounding them or a parenchymal beak. Examples include lymphadenopathy, branchial cysts, schwannoma, lipomas, neurofibromas, and masseter muscle pathology (Figures 5-9).All these were easily recognised and characterised on US except for the schwannoma, where US could only diagnose an extraparotidlesion, possibly of mesenchymal origin. US guided FNAC confirmed the diagnosis.

\section{Intraparotid lesions}

Intraparotid lesions usually show a visible rim of surrounding parenchyma on US or a beak sign. Retention cysts may occur secondary to inflammation or obstruction. They appear anechoic, cystic and well defined. Enlargement of intraparotid lymph nodes are well demonstrated on US and are normal in children. In adults, one has to consider viral infections like infectious mononucleosis (IMS) and cytomegalovirus (CMV), metastases, Sjögren syndrome and lymphoma.

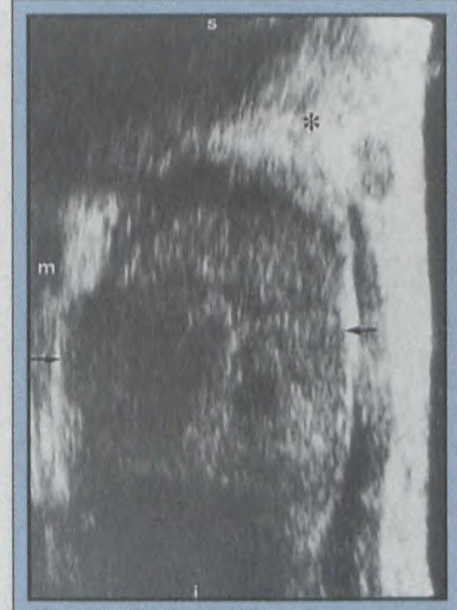

Figure 5: Extraparotid lymphadenopathy. Longitudinal sonogram of a patient with LettererSiwe disease shows a large solid malignant /ymph node of the upper cervical chain (arrows) abutting the parotid (asterisk). Metastatic lymphadenopathy in the jugulodigastric region, particularly from a primary headand neckmalignancy, can also present as a parotid swelling.

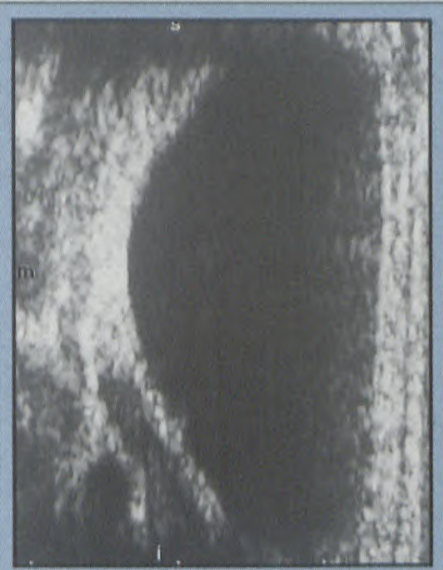

Figure 6: Branchial cleft cyst - an embryological remnant of the second branchial cleft. Longitudinal sonogram of the lateral cenvical region shows a large echo-free cyst with smooth inner walls.

\section{Neoplasms}

Eighty per cent of salivary gland tumours are found in the parotid gland, $80 \%$ of parotid tumours are benign and $80 \%$ occur in the superficial lobe. ${ }^{2}$ The commonest benign neoplasms are the pleomorphic adenoma and the adenolymphoma (Warthin's cyst). Although the pleomorphic adenoma is more common in females and the

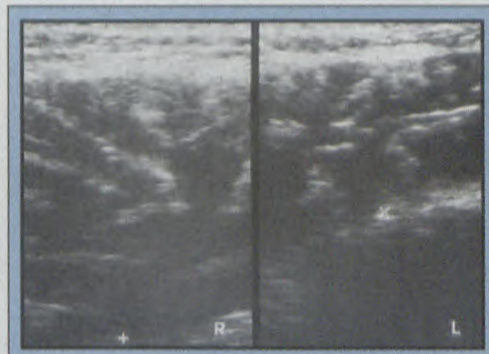

Figure 7: Massetermuscle hypertrophy This is often idiopathic and occurs in adolescence and young adulthood. It can be unilateral or bilateral. Axial images of both massetermusclesshowa marked discrepancy in thickness. The right masseterishypentrophied.

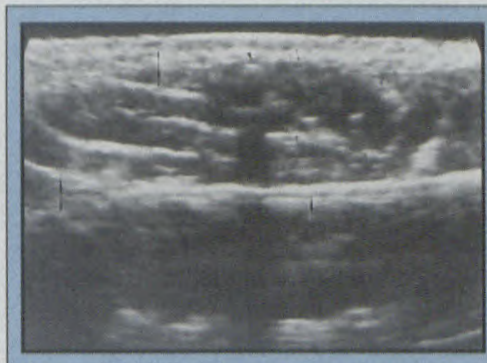

Figure 8: Hemangioma of the masseter muscle. Longitudinal (coronal) image shows two echogenic phleboliths with posterior acoustic shadowing (arrowheads) within the masseter muscle at the angle of the jaw.

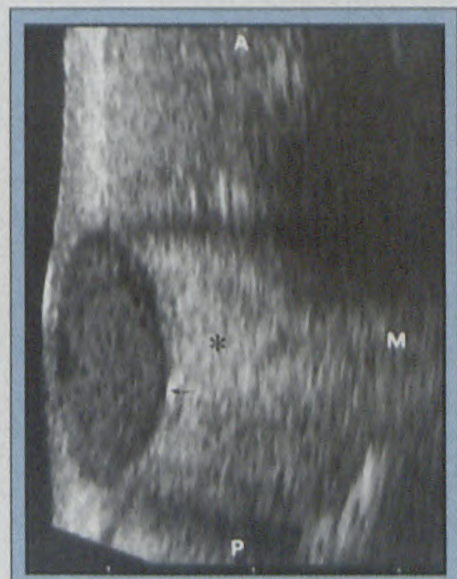

Figure 9: Schwannoma. Axial scanon the left shows a lobulated, well defined, solid, hypoechoic nodule (arrows) abutting the parotid (asterisk) in a patient with neurofibromatoses. FNA confimedaschwannoma.

Warthin's cyst more common in males these tumours are often not easily distinguishable on US.They appear as homogenous, hypoechoic, well defined, occasionally lobulated or cystic masses, and may show posterior acoustic 
Irom page 19

enhancement(Figure 10).Pleomorphic adenomas may recur, and reportedly undergo malignant transformation in $2-5 \%{ }^{2.3}$. Warthin's cyst occurs virtually only in the parotid gland ${ }^{4}$, usually in the tail region. They arise from heterotopic salivary ductepithelium in

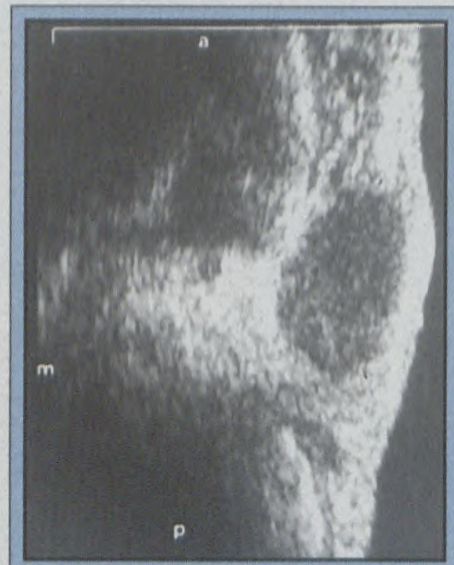

Figure 10: Pleomorphicadenoma. Axial sonogram shows a well defined, hypoechoic, homogenouslesion (amows), with posterior through transmission of sound.

the intraparotid lymph nodes, and hencecan bemulticentric (30-95\%) and bilateral $(30 \%)^{5}$.

Malignant neoplasms are distinctly uncommon in the parotid. They account for less than $6 \%$ of salivary neoplasms and are more common in the minor salivary glands. The commonest include mucoepidermoid carcinoma (Figure 11), adenoid cystic

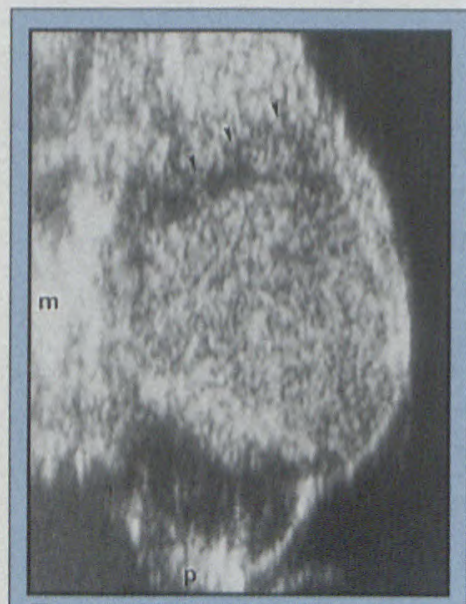

Figure 11: Mucoepidemoid carcinoma Axialsonogram showsa heterogeneous solidmass lesion (arrows) with areas of necrosis and relatively well defined margins (a known feature). A rim of residual nomal parotid remains (arrowheads).

carcinoma, malignant pleomorphic adenoma, lymphoma and metastases (e.g. squamous cell carcinoma, melanoma).US features of a malignant neoplasm are a hypoechoic, heterogeneous mass lesion with

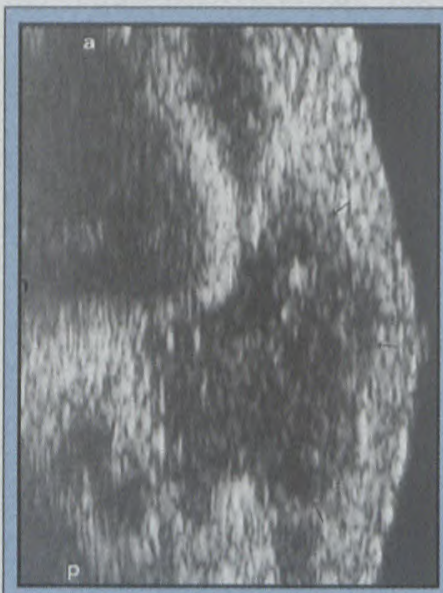

Figure 12: Adenocarcinoma of the parotid.

Animegular, hypoechoic, inhomogenous lesion is seen involving both superficial and deep lobes of the parotidon transverse US. Itsmarginsare irregular and poorly defined.

irregular, ill defined margins, and infiltration of adjacent tissues (Figure 12). Symptoms of pain and paraesthesia, facial nerve palsy, rapid increase in size and cervical lymphadenopathy should raise the suspicion of a more sinister lesion.

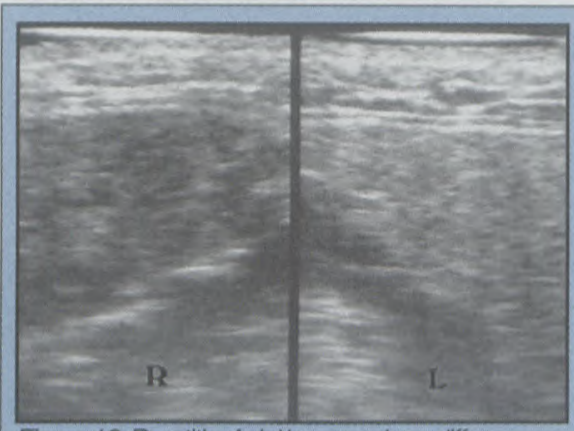

Figure 13: Parotitis. Axial images show diffuse marked homogenous hypoechogenicity of the right parotid compared to the left.

\section{Inflammation}

Inflammatory parotidenlargement can be secondary to:(1) Viral infection, e.g. mumps; (2) Bacterial parotitis,

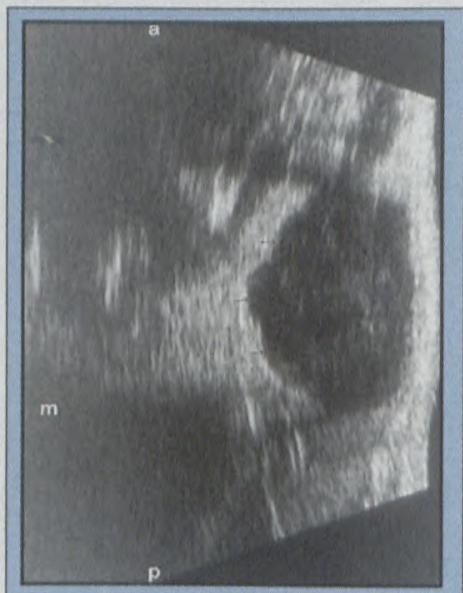

Figure 14: Parotidabscess. Longitudinal USshows a focal, hypoechoic, mass lesion (arrow heads) with ill defined posteriormargin (arrow) in a patient whopresented with a painful, tender parotidmassandfever. especially in diabetics, debilitated and post surgical patients; (3) Granulomatous conditions, eg. tuberculosis, sarcoidosis; (4) Immune parotitis, particularly Sjögren syndrome.

Mumps parotitis causes diffuse, bilateral swelling of the parotids, which can be asynchronous (Figure 13). There is often associated cervical lymphadenopathy. Parotid abscess is seen on US as a focal, hypoechoic mass lesion that can be sharply circumscribed or have poorly defined margins, and can infiltrate beyond the parotid gland (Figure 14). Clinical correlation is vital in confirmation of an abscess. Immune parotitis is best illustrated by Sjögren syndrome which is a benign, chronic, systemic autoimmune disorder causing destruction of acinar tissue in exocrine glands. It occurs primarily in women aged 40 to 60 years, and presents classically with a triad of xerostomia, keratoconjunctivitis sicca and an associated connective tissue disorder, most commonly rheumatoid arthritis. Typical sialographic features are punctate spherical collections of contrast noted throughout the parotid gland.US demonstrates bilateral diffuse parotid enlargement with reticulated hypoechogenicity, with or without cystic change (Figures 15A,B,C).

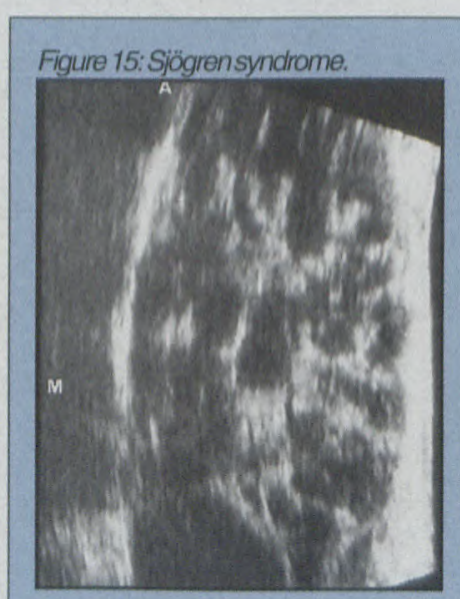

A. Transverse US showsmultiplecystic and hypoechoicareas throughout the parotidgland. Nonomal parenchymais
seen.

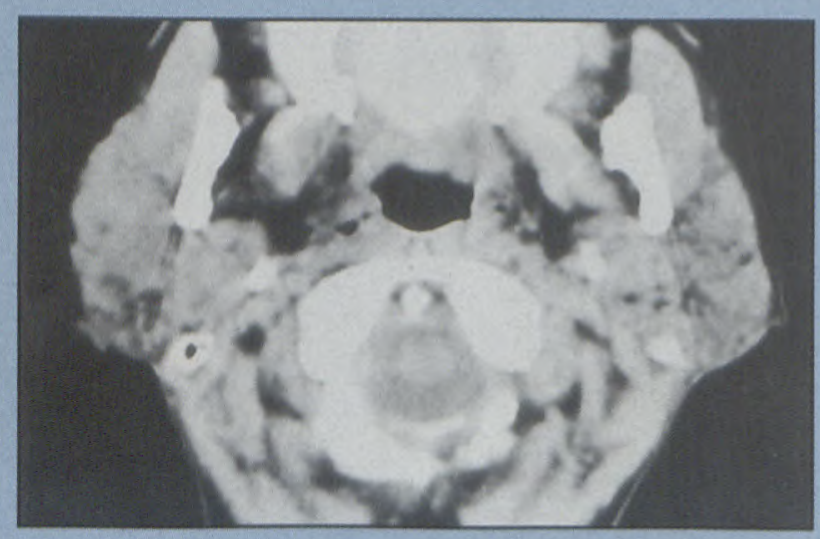

B. AxialCT of the same patient shows bilateral symmetrical enlargerment of the parotid gland, demonstrating soft tissue and low density areas within. 


\section{The backbone of}
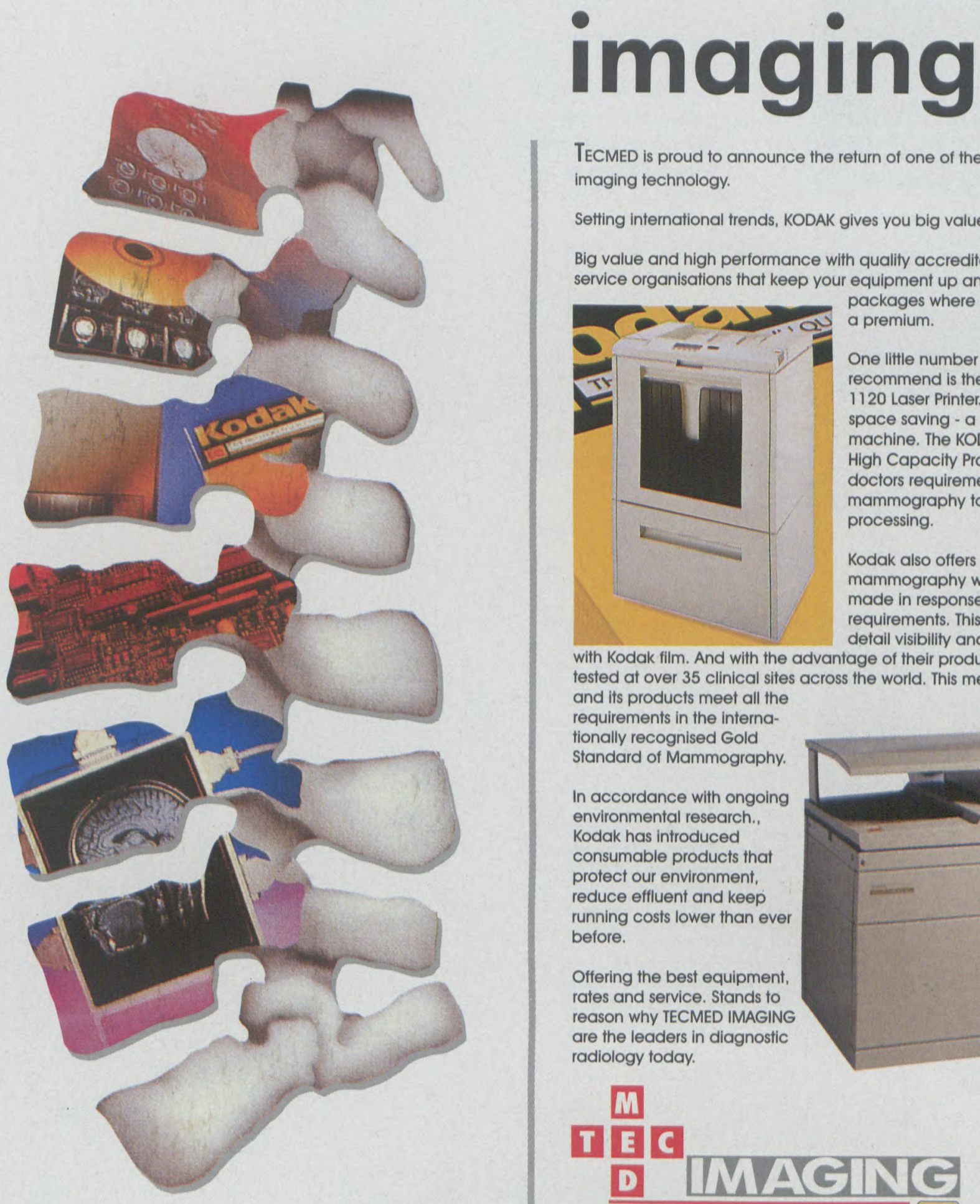

TECMED is proud to announce the return of one of the world's specialists in imaging technology.

Setting international trends, KODAK gives you big value with small packages.

Big value and high performance with quality accredited marketing and service organisations that keep your equipment up and running. Small

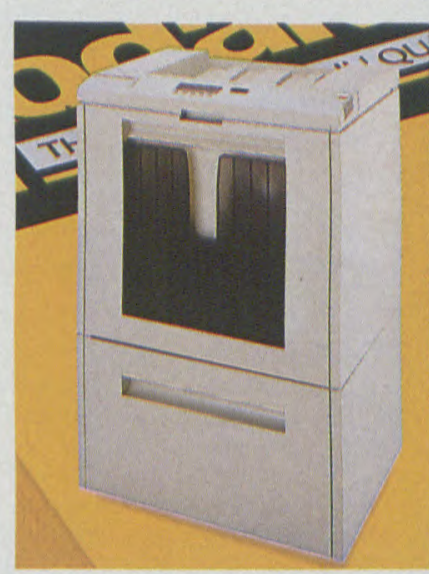

packages where space and costs are at a premium.

One little number that we'd like to recommend is the KODAK 'EKTASCAN' 1120 Laser Printer. It's compact, neat and space saving - a big number for a small machine. The KODAK X-OMAT 270 RA High Capacity Processor meets all doctors requirements from mammography to 45 second rapid processing.

Kodak also offers the best technology in mammography with improvements made in response to customer requirements. This means better detail visibility and sharpness with Kodak film. And with the advantage of their products being tested at over 35 clinical sites across the world. This means Kodak and its products meet all the requirements in the internafionally recognised Gold Standard of Mammography.

In accordance with ongoing environmental research., Kodak has introduced consumable products that protect our environment, reduce effluent and keep running costs lower than ever before.

Offering the best equipment, rates and service. Stands to reason why TECMED IMAGING are the leaders in diagnostic radiology today.
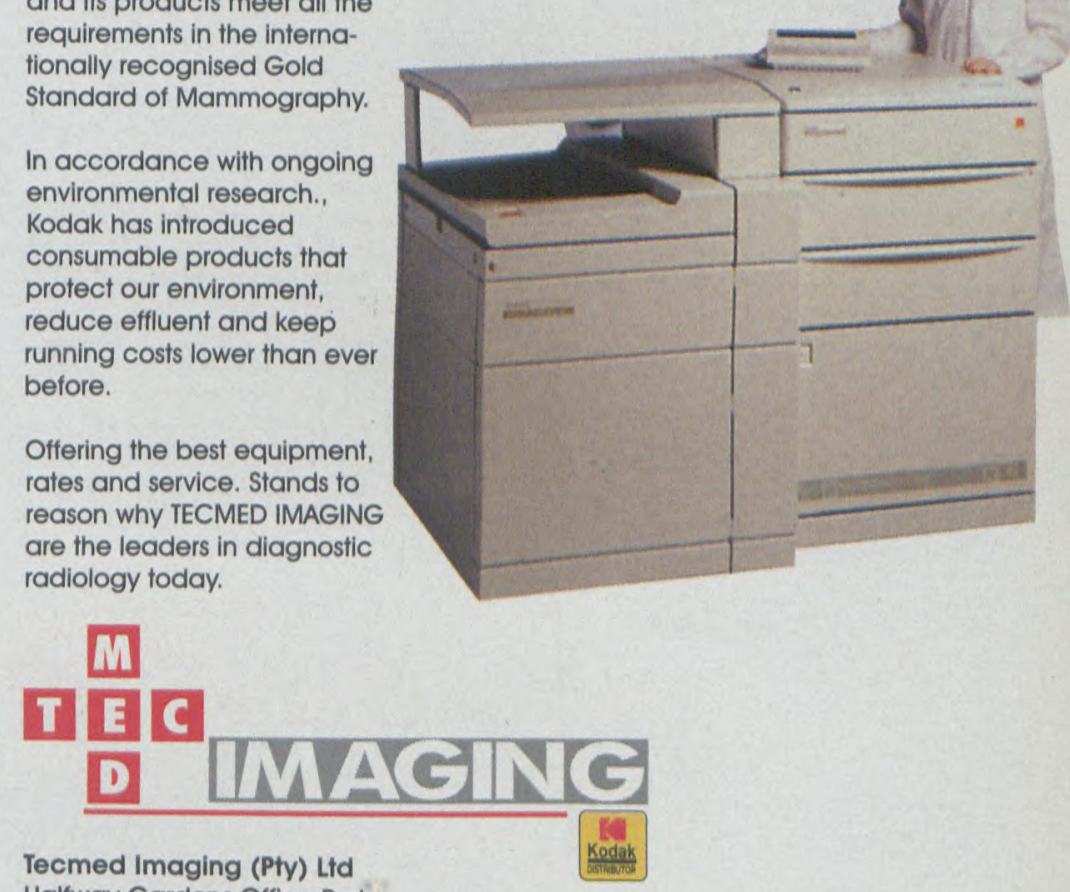

Halfway Gardens Office Park,

Asparagus Road, Vorna Valley, Midrand.

P.O. Box 4229, Halfway House 1685.

Tel: (011) 315-4874

Durban: (031) 701-0311

Cape Town: (021) 592-2464/5/6/7

Bloemfontein: (051) 30-4211 


\section{Role of Ultrasound in the Imaging of Parotid swellings}

\section{from page 20}

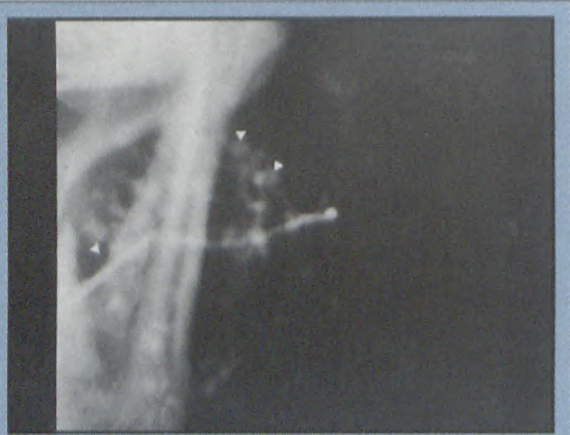

15C. Sialogram shows mild to moderate sialectasia. Spherical collections of contrast medium (arrowheads) are noted throughout the gland.

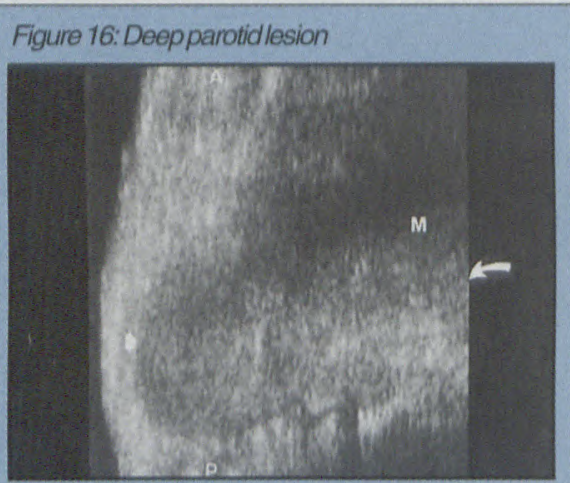

A.Axial US showsan extensive, solid mass lesion involving the deep parotid (shortarrow) without adequate delineation of the inner/medial margins (curvedarrow).

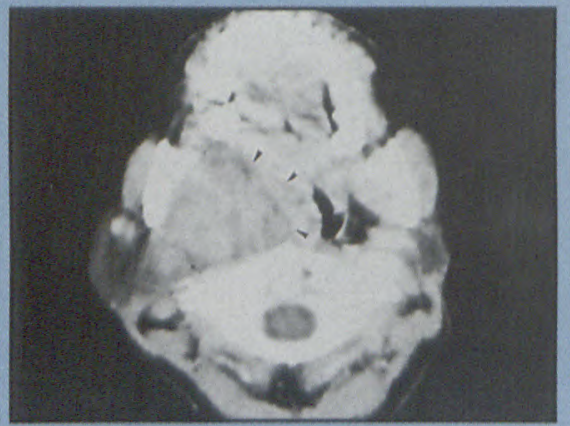

B. Transverse CT reveals a massivelesion arising from the oropharyngeal region (amowhead) and infiltrating the parotid gland (open shortarrow). Biopsy showed oropharyngeal adenocarcinoma.
Associated lymphadenopathy should alert one to the possible complication of lymphoma, which signals a grave prognosis and is often rapidly fatal ${ }^{6}$.

\section{Conclusion}

US remains an inexpensive, rapid and non-invasive technique for imaging of the parotid. Its ability to predict the histologic nature of a focal mass lesion may be further enhanced by US guided $\mathrm{FNAC}^{7}$. The major limitations of US are in the evaluation of deep lobe parotid lesions (Figure 16A,B), parapharyngeal extension and skull base destruction, when $\mathrm{CT}$ is the imaging modality of choice.Although magnetic resonance imaging offers excellent contrast resolution and multiplanar facilities in the delineation of parotid lesions, it is as yet equally unreliable in the prediction of the histology of a mass lesion ${ }^{8}$, still not widely available and relatively expensive.

\section{References}

1. Handbooks in Radiology: Headand Neck Imaging N. Ric Hamsberger Year Book

Medical Publishers, Inc., St Lovis Mosby-Year Book, Inc, 1990:248Chicago, London. 2. Batsakis JG. Tumours of the head and neck, 2nded. Baltimore:Williams \&'Wilkins, 1979: 1.20

3. Som PM, Shugar IM, Sacher M, et al. Benign and malignant parotid pleomorphic adenomas: CT and MR studies, JCAT 1988; 12:65. 4. BeckLD, MagndaTA. Papillary cystadenoma hymphomatosum (Warthin's tumour): a multicentric benign tumour, Lanyngosope 1967;77:1840.

5. Chaudhry AP, Gorlin RP.Papillary cystadenomalymphomatosum (adenolymphoma),AmJSung 1958;95:723. 6. Rankow RM, Polayes IM. Diseases of the Salivary Glands Philadelphia:WBSaunders, 1976:202:228,284303.

7. Teresi LM, Lufkin RB, Wortham DG, etal. Parotid masses:MR imaging Radiology 1987; 163:405-409.

8. Freling NJM, MolenaarWM, Vermey A, et al. Malignant parotid tumours: Clinical use of MR imaging and histologiccorrelation, Radiology 1992; 185:691-696. 